\title{
Cyclophosphamide augments the anti-tumor efficacy of uracil and tegafur by inhibiting dihydropyrimidine dehydrogenase
}

\author{
YOSHINORI NIO ${ }^{1}$, CHIKAGE IGUCHI ${ }^{1}$, HIROSHI KODAMA ${ }^{1}$, MASAYUKI ITAKURA ${ }^{2}$, KOJI HASHIMOTO ${ }^{2}$, \\ MAKOTO KOIKE $^{2}$, TOMOKO TOGA ${ }^{2}$, RIRUKE MARUYAMA ${ }^{3}$ and MASAKAZU FUKUSHIMA ${ }^{4}$ \\ ${ }^{1}$ Kodama Breast Clinic, Kyoto 603-8325; Departments of ${ }^{2}$ Digestive and General Surgery; ${ }^{3}$ Clinical Pathology, \\ Shimane University School of Medicine, Izumo 693-8501; ${ }^{4}$ Tokushima Research Center, \\ TAIHO Pharmaceutical Co. Ltd., Tokushima 771-0194, Japan
}

Received May 12, 2006; Accepted August 21, 2006

\begin{abstract}
The present study assesses the effects of neoadjuvant chemotherapy (NAC) with uracil and tegafur (UFT) alone vs UFT plus cyclophosphamide (CPA), on the activity of thymidylate synthase (TS) and dihydropyrimidine dehydrogenase (DPD) in breast cancer tissues. Breast cancer patients were randomly assigned to 3 groups; the control (no-treatment) group $(n=13)$, the UFT $(5-8 \mathrm{mg} / \mathrm{kg} /$ day $)$ alone group $(n=10)$ and the UFT plus CPA (1 mg/kg/one day interval) (UC) group $(n=9)$, and they received NAC for 2-4 weeks. A total of 32 invasive ductal breast carcinomas were used to assay for TS and DPD activity. There were no statistically significant differences in tumor size or stage classification between the 3 groups. The DPD activity was inversely and significantly correlated with the tumor size and pT, but the TS activity was not correlated with these clinicopathological factors. The TS activity was decreased by NAC with UFT, and the addition of CPA resulted in an increased inhibition of TS activity. In contrast, DPD activity was increased by NAC with UFT administration, but its increased activity was significantly inhibited by the addition of CPA. Multiple regression analyses demonstrated that the total dose of UFT was a significant variable for inhibiting TS activity, and that CPA was a significant variable for inhibiting DPD activity. The DPD activity increased by UFT can be inhibited by CPA, and this may represent one of the possible mechanisms responsible for the anti-tumor activity of 5-FU or its derivatives as enhanced by CPA.
\end{abstract}

\section{Introduction}

Cyclophosphamide (CPA) is widely used for chemotherapy against breast cancer in combination with adriamycin (ADR),

Correspondence to: Dr Yoshinori Nio, Kodama Breast Clinic, 35 Kitano-Kamihakubai-cho, Kita-ku, Kyoto 603-8325, Japan

E-mail: kodama-breast-clinic@theia.ocn.ne.jp

Key words: breast cancer, uracil and tegafur (UFT), cyclophosphamide, thymidylate synthase, dihydropyrimidine dehydrogenase methotrexate (MTX) or 5-fluorouracil (5-FU). We are interested in the combination effects of CPA with 5-FU or its derivatives, because CPA frequently shows remarkable augmenting effects on the anti-tumor effects of 5-FU or its derivatives.

In Japan, instead of intravenous (iv) 5-FU, an oral fluoropyrimidine, uracil-tegafur (UFT), has been widely used for the treatment of various malignancies (1). UFT is as effective as a continuous infusion of 5-FU, but with a better toxicity profile $(2,3)$. Furthermore, one particular pharmacokinetic study demonstrated that the 5-FU concentration in the blood after oral UFT was comparable to that after a continuous iv infusion of 5-FU (4).

Several previous clinical reports have suggested that the combination regimen of UFT+CPA (UC) showed a good antitumor activity against breast cancer, sometimes in combination with anthracyclines $(5,6)$. We have also used oral chemotherapy with UFT plus oral CPA (oral UC regimen) for adjuvant chemotherapy after pancreatic cancer surgery, and a retrospective analysis demonstrated that this UC regimen was beneficial in improving survival after pancreatic cancer surgery (7). Furthermore, there were also some patients, whose recurrent pancreatic or ampullary carcinomas responded to oral UC therapy $(8,9)$.

Although it has been suggested that CPA can augment the antitumor activity of 5-FU or its derivatives, the mechanisms responsible remain unclear. However, several reports have suggested that $\mathrm{CPA}$ can augment the antitumor activity of 5-FU derivatives by modulating the activity of pyrimidine-metabolizing enzymes. It has also been reported that CPA augmented the activity of ribonucleotide reductase (RNR), which metabolizes 5-FU to fluoro-deoxyuridine monophosphate (FDUMP) (10); other researchers have also reported that $\mathrm{CPA}$ enhanced the efficacy of capecitabine and 5'-deoxyfluorouridine, which are also 5-FU derivatives, by inducing thymidine phosphorylase (TP) in a human breast tumor xenograft model (11). Although these modulatory effects of CPA on RNR or TP may represent some of the mechanisms responsible for the effects of CPA on the anti-tumor activity of 5-FU or its derivatives, no studies to date have investigated the effects of CPA on the key enzymes in pyrimidine metabolism, 
thymidylate synthase (TS) and dihydropyrimidine dehydrogenase (DPD).

TS is an enzyme, which catalyzes the reductive methylation of 2'-deoxyuridine-5'-monophosphate (dUMP) by 5,10methylene-5,6,7,8-tetrahydrofolate $\left(\mathrm{CH}_{2} \mathrm{H}_{4}\right.$ folate) to produce thymidine-5'-monophosphate (dTMP) and 7,8-dihydrofolate $\left(\mathrm{H}_{2}\right.$ folate). TS is a dimer of identical $30-35 \mathrm{kDa}$ subunits, and two major pathways are known in the metabolism of pyrimidine during DNA synthesis, the de novo pathway and the salvage pathway. TS is a key enzyme in the de novo pathway of pyrimidine synthesis, and is a very important target for chemotherapy with 5-FU or its derivatives (12).

DPD degrades fluoropyrimidine to 2-fluoro- $\beta$-tetrahydrofolate $\left(5,10-\mathrm{CH}_{2} \mathrm{FH}_{2}\right)$, and is the initial and rate-limiting enzyme in this process. Fluoropyrimidines are degraded by DPD mainly in the liver, but the DPD activity in the tumor is correlated with the clinical response to 5-FU-based chemotherapies $(13,14)$.

The present study was designed to assess the effects of neoadjuvant chemotherapy (NAC) with UFT alone vs UFT plus CPA (UC regimen) on the activity of TS and DPD in human breast cancer tissues.

\section{Patients and methods}

Patient enrolment. The study included 32 invasive ductal carcinomas of the breast. Two basic criteria had to be met before NAC administration: i) Histological or cytological proof of breast cancer and a preoperative diagnosis that the tumor could be curatively resectable, and ii) a performance status (PS) of 0-3 (ECOG score). The contraindications included: i) Total disability ( $\mathrm{PS}=4, \mathrm{ECOG}$ score), ii) prior chemotherapy, radiotherapy or immunotherapy within 4 weeks, iii) an active infectious disease, iv) severe anemia (hemoglobin $<9 \mathrm{~g} / \mathrm{dl}$ ), leukopenia $\left(<3,000 / \mathrm{mm}^{3}\right)$, thrombocytopenia $\left(<70,000 / \mathrm{mm}^{3}\right)$, azotemia (creatinine $>2 \mathrm{mg} / \mathrm{dl}$ ), or liver dysfunction (GOT, GPT and alkaline phosphatase $>4$-fold the normal limits), v) severe heart disease or a concomitant malignant disease, and vi) pregnancy.

All patients and their families in the treatment group were fully informed with regard to the study aim, treatment program, expected side-effects and clinical benefits of the study, and informed consent was obtained in all cases. After surgery, the stage of breast cancer was classified according to the UICC (TNM) stage classification system (15).

Neoadjuvant chemotherapy (NAC). With their informed consent, the breast cancer patients were randomly assigned into 3 groups: The control (no-treatment) group, the UFT alone group and the UFT plus CPA (UC) group. The CPA alone group was not set-up, because in Japan, under the universal health insurance system, the Japanese Ministry of Health, Labor and Welfare strictly regulates the use of anticancer agents, and they do not approve of chemotherapy with CPA alone against breast cancer. UFT was orally administered at $5-8 \mathrm{mg} / \mathrm{kg} /$ day daily, and CPA was orally administered at $50 \mathrm{mg} /$ body every other day. The NAC usually started from the day of the first visit to the outpatient ward until 2 days before the surgery for 2-4 weeks. The administration period was dependent on the waiting period for surgery. The dose of UFT is usually expressed as the dose of FT and the dose of UFT was set at $8 \mathrm{mg} / \mathrm{kg} /$ day for the patients under 70 years of age, and at $6 \mathrm{mg} / \mathrm{kg} / \mathrm{day}$ for those over 71 years old, but it was difficult to administer an accurate dose in terms of $/ \mathrm{kg}$ or $/ \mathrm{m}^{2}$. UFT is an oral agent and is usually administered in a capsule or granule package, and one capsule contains $100 \mathrm{mg}$ UFT whereas one granule package contains $150 \mathrm{mg}$ UFT.

Evaluation of side-effects. In the patients in the NAC groups, hematology, serum biochemistry, tumor markers, the symptomatic status and PS, were examined before surgery routinely at biweekly intervals, or sometimes more frequently. Toxicity was evaluated according to the National Cancer Institute - Common Toxicity Criteria (16). If toxicity occurred, then the first CPA administration was interrupted and the dose of UFT for each patient was decreased according to the patient's condition (PS, body weight, age, hematology and serum biochemistry).

Evaluation of objective response (OR). If possible, the size of the primary lesion was assessed by ultrasonography (USG) or mammogrphy (MMG) before and after NAC administration (usually 1 or 2 days before surgery). The response was evaluated by the RECIST (unidimensional) guideline (17). In brief, the largest diameter of the lesion was taken from bidimensional measurements. The responses were categorized as follows: i) A complete response (CR) indicating a complete disappearance of the lesion, ii) a partial response (PR) indicating a $>30 \%$ decrease in tumor diameter, iii) progressive disease (PD) indicating a $>20 \%$ increase in tumor diameter, or iv) stable disease (SD) (neither CR, PR nor PD).

If the tumor was evaluated to be progressive, then NAC was suspended. The duration of the response was not included in the evaluation of the OR, because all patients underwent surgery.

Pathological response. The histopathological effect was evaluated based on the grade (0-3), according to the histopathological criteria for the assessment of therapeutic response in breast cancer by the Japanese Breast Cancer Society (18). In brief, grade 3 (complete response) represented the necrosis or disappearance of all tumor cells, and the replacement of all tumor cells by granuloma-like and/or fibrous tissue. In the case of a complete disappearance of the tumor cells, pretreatment pathological evidence of the presence of a tumor is necessary; grade 2 (marked response) represented marked changes in two thirds or more of the tumor cells; grade 1 (slight response); grade 1a (mild response) represented mild changes in the cancer cells regardless of the area, or marked changes seen in less than one third of the cancer cells; grade $1 \mathrm{~b}$ (moderate response) represented marked changes in one third or more but less than two thirds of the tumor cells; and grade 0 (no response) represented almost no changes in the tumor cells after treatment.

Measurement of TS and DPD activity in tissues. The tissues were homogenized with 3 volumes of $50 \mathrm{mM}$ Tris- $\mathrm{HCl}$ (pH 7.6) containing $10 \mathrm{mM}$ 2-mercaptoethanol, $25 \mathrm{mM} \mathrm{KCl}$ and $5 \mathrm{mM} \mathrm{MgCl}$, centrifuged at $105,000 \mathrm{~g}$ for $60 \mathrm{~min}$, and 
Table I. Backgrounds of the patients.

\begin{tabular}{lccc}
\hline & $\begin{array}{c}\text { Control } \\
(\mathrm{n}=13)\end{array}$ & $\begin{array}{c}\text { UFT } \\
(\mathrm{n}=10)\end{array}$ & $\begin{array}{c}\text { UFT+CPA } \\
(\mathrm{n}=9)\end{array}$ \\
\hline Age & $52.3 \pm 8.8^{\mathrm{a}}$ & $56.6 \pm 14.4$ & $64.7 \pm 13.8^{\mathrm{a}}$ \\
Clinical stage & $2.22 \pm 0.99$ & $2.30 \pm 0.75$ & $2.19 \pm 0.71$ \\
Tumor size (cm) & $2.55 \pm 1.23$ & $2.89 \pm 1.85$ & $3.20 \pm 1.64$ \\
pT & $1.69 \pm 0.75$ & $1.80 \pm 0.63$ & $2.00 \pm 0.71$ \\
pN & $0.92 \pm 1.19$ & $0.90 \pm 0.88$ & $0.56 \pm 1.01$ \\
Number of & $3.77 \pm 6.70$ & $2.80 \pm 5.77$ & $3.22 \pm 8.93$ \\
involved nodes & & & \\
ER status (+:-) & $10: 3$ & $8: 2$ & $6: 3$ \\
Administration & & $26.7 \pm 6.5$ & $26.1 \pm 8.4$ \\
period (days) & & & \\
Total dose of & & $9.09 \pm 3.10$ & $8.28 \pm 3.51$ \\
UFT (g) & & & $653 \pm 211$ \\
Total dose of & & & \\
CPA (mg) & & & \\
\hline
\end{tabular}

${ }^{\mathrm{a}} \mathrm{p}=0.0180$.

then the resulting supernatant was used to measure the enzyme activity.

TS activity was measured by the [6-3H]-FdUMP-binding assay based on the method of Spears et al (19).

DPD was assayed, by a modification of the method of Naguib et al (20) as described previously (21). Briefly, a reaction mixture containing $10 \mathrm{mM}$ potassium phosphate (pH 8.0), 0.5 mM EDTA, $0.5 \mathrm{mM}$ 2-mercaptoethanol, $2 \mathrm{mM}$ dithiothreitol, $5 \mathrm{mM} \mathrm{MgCl} 2,20 \mu \mathrm{M}\left[6-{ }^{14} \mathrm{C}\right]-5-\mathrm{FU}, 0.1 \mathrm{mM}$ $\mathrm{NADPH}$, and $25 \mu \mathrm{l}$ of the enzyme extract in a total volume of $50 \mu \mathrm{l}$ was incubated at $37^{\circ} \mathrm{C}$ for $30 \mathrm{~min}$. The DPD activity was determined as the sum of the products formed from 5-FU: Dihydrofluorouracil (DHFU), 2-fluoro- 3 -ureidopropionic acid, and 2-fluoro- $\beta$-alanine. After the addition of $25 \mu 1$ $0.36 \mathrm{mM} \mathrm{KOH}$, the reaction mixture was allowed to stand at room temperature for at least $30 \mathrm{~min}$ to hydrolyze the DHFU formed, and was then neutralized with $25 \mu 10.36 \mathrm{mM} \mathrm{HClO}_{4}$ and immediately centrifuged at $14,000 \mathrm{rpm}$ for $10 \mathrm{~min}$. A $5 \mu \mathrm{l}$ aliquot of the supernatant was applied to a silica gel 60F254 plate $(2.5 \times 20 \mathrm{~cm}$, Merck, Darmstadt, Germany), and developed with a mixture of methanol and $1 \mathrm{M}$ ammonium acetate $(5: 1, v / v)$. Each product was visualized and quantified using an imaging analyzer (BAS-2000, Fujix, Tokyo).

Statistics. The Chi-square test and Student's t-test were used for comparison of the patients' clinicopathological backgrounds. The correlation between enzyme activity and clinicopathological factors was examined using Pearson's correlation analysis. A p-value of $<0.05$ was considered to be statistically significant.

\section{Results}

The background data of the 3 groups are summarized in Table I. The age of the UC group was significantly higher than the control group. Although the tumor size in the UC
Table II. Objective and pathological responses.

\begin{tabular}{lcc}
\hline \multicolumn{3}{c}{ Objective response (RECIST) } \\
\cline { 2 - 3 } & UFT & UFT+CPA \\
\hline \% inhibition (mean \pm SD) & $12.8 \pm 8.9$ & $11.2 \pm 13$ \\
CR or PR & 0 & 1 \\
SD & 8 & 7 \\
PD & 0 & 1 \\
Not evaluated & 2 & 0 \\
Total & 10 & 9 \\
& Pathological response & \\
Grade & & 0 \\
1b & 1 & 8 \\
la & 7 & 1 \\
0 & 2 & 9 \\
Total & 10 & \\
\hline
\end{tabular}

group was the largest among the 3 groups, there were no significant differences in tumor size or clinical stage among the 3 groups.

The ORs and pathological responses are summarized in Table II. NAC with UFT or UC resulted in an average 10\% decrease in unidimensional tumor size, and there were no differences in the percentage decrease in tumor size between the two groups. One PR was observed in the UC group, and the changes in the MMG are shown in Fig. 1. The pathological responses were evaluated as $1 \mathrm{a}$ or $1 \mathrm{~b}$ in most cases.

The TS and DPD activity is summarized in Fig. 2. The TS activity was highest in the control group, followed by the UFT and UC groups, and the TS activity in the UC group was significantly lower than that in the control group $(\mathrm{p}=0.0230)$. In contrast, the DPD activity was highest in the UFT group, followed by the control and UC group, and the DPD activity in the UC group was significantly lower than that in the UFT group $(\mathrm{p}=0.0355)$.

The correlations between the TS or DPD activity with the clinicopathological factors are summarized in Table III. The DPD activity was inversely correlated with the tumor size and $\mathrm{pT}$, and these correlations reached statistical significance ( $p=0.0108$ and 0.0051 , respectively). The DPD activity was also inversely correlated with ER expression, but this was only a statistical trend $(\mathrm{p}=0.086)$. In contrast, the TS activity was not correlated with these clinicopathological factors. Furthermore, multiple regression analyses indicated that the total dose of UFT administered and the administration period were significant variables for the TS activity, and that the total dose of CPA administered and the administration period, as well as $\mathrm{pT}$, were significant variables for the DPD activity (Table IV).

\section{Discussion}

The present study did not aim to produce CRs or pCRs by NAC with UFT alone or the UC regimen. The OR in the present study was SD and the pathological responses were 

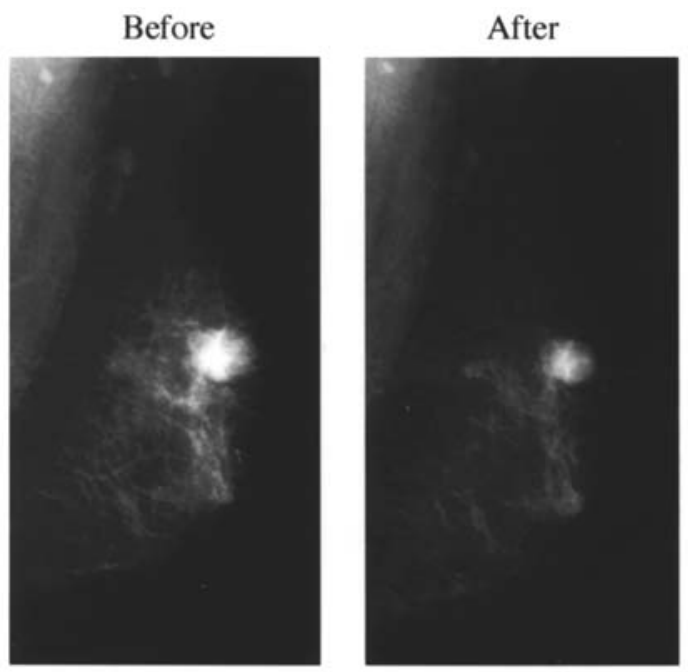

Medio-lateral-oblique position
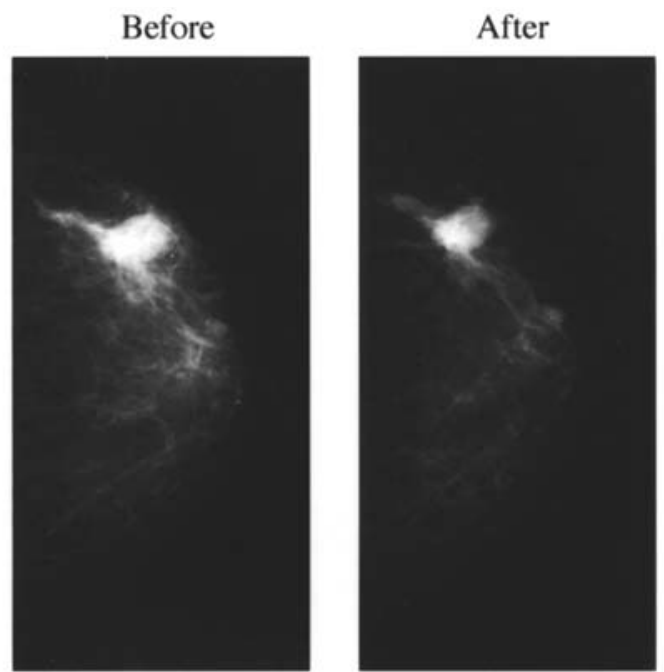

Cranio-caudal position

Figure 1. Comparative mammography before and after NAC with UFT+CPA in a patient with breast cancer (55-year-old female, invasive ductal carcinoma). The patient received UFT at $300 \mathrm{mg}$ /day daily and CPA at $50 \mathrm{mg}$ at one day intervals for 28 days. The tumor size decreased from $29 \mathrm{~mm}$ to $20 \mathrm{~mm}$ in longitudinal diameter (30\% decrease), which was evaluated as a PR according to the RECIST guideline.

TS activity (pmol/mg protein)

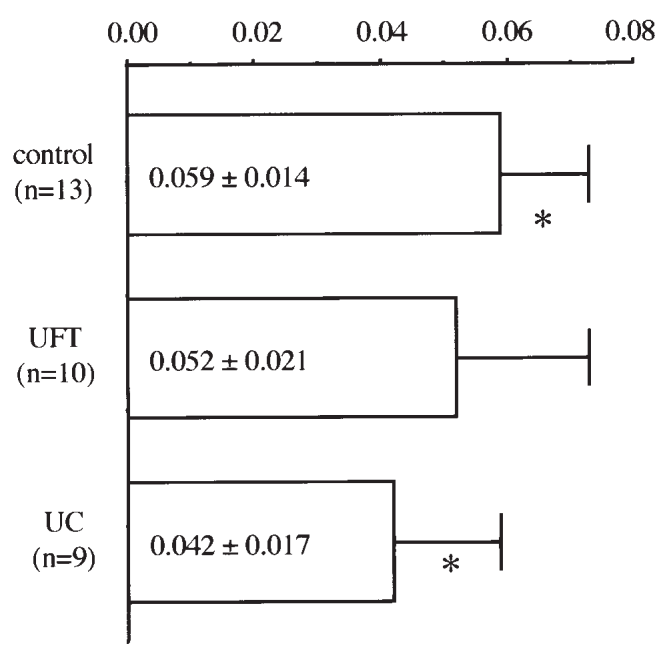

DPD activity (pmol/min/mg protein)

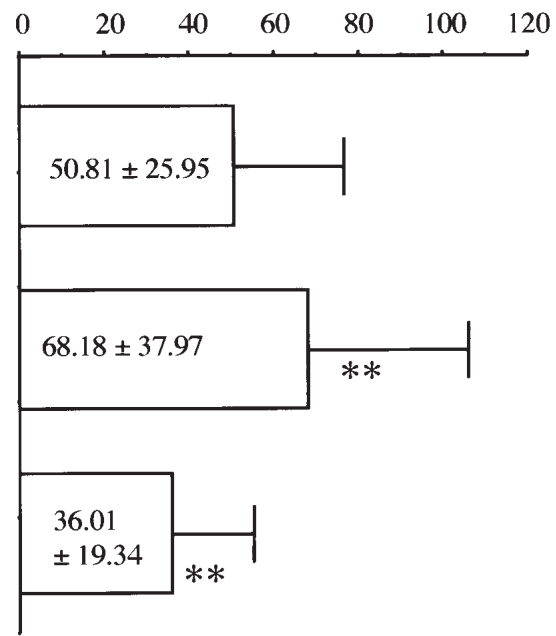

Figure 2. Comparative activities of TS and DPD after NAC with UFT or the UC regimen. The values represent means \pm SD. The cancer group included the control, UFT and UC groups. ${ }^{*} \mathrm{p}=0.0230,{ }^{* *} \mathrm{p}=0.0355$.

Table III. Correlation between TS or DPD activity and clinicopathological factors.

\begin{tabular}{lcc}
\hline Variables & TS (p-value) & DPD (p-value) \\
\hline DPD & $-0.078(0.6729)$ & - \\
Age & $-0.038(0.8392)$ & $0.087(0.6387)$ \\
Clinical stage & $-0.059(0.7507)$ & $-0.166(0.3677)$ \\
Tumor size & $-0.132(0.4742)$ & $-0.441(0.0108)$ \\
pT & $-0.080(0.6666)$ & $-0.478(0.0051)$ \\
pN & $-0.017(0.9260)$ & $0.082(0.6575)$ \\
ER expression & $0.040(0.8310)$ & $-0.327(0.0674)$ \\
\hline
\end{tabular}

The values indicate $r$-values and the parentheses indicate $\mathrm{p}$-values. slight in most cases. These results suggest that, in the present study, the administration dose might be too low and the administration period might be too short to induce an obvious OR against breast cancer, because a previous study in gastric cancer demonstrated that NAC with oral UFT resulted in a $33.3 \% \mathrm{OR}$, and the mean totally administered dose of UFT was $7.67 \mathrm{~g}$, respectively, and the mean administration period was 20.8 days (22). In the present study, the mean totally administered doses of UFT were $9.09 \mathrm{~g}$ in the UFT group and $8.28 \mathrm{~g}$ in the UC group, and the mean administration periods were 26.7 and 26.1 days, respectively (Table I). Accordingly, in order to induce an obvious OR in breast cancer, UFT should be administered in larger doses and for longer periods. The present study aimed to assess the changes in intratumoral TS or DPD activities after NAC, and 
Table IV. Multiple regression analysis on TS and DPD activities.

\begin{tabular}{lcccc}
\hline \multirow{2}{*}{ Variables } & \multicolumn{2}{c}{ TS activity } & \multicolumn{2}{c}{ DPD activity } \\
\cline { 2 - 5 } & $\mathrm{r}$ & $\mathrm{p}$-value & $\mathrm{r}$ & $\mathrm{p}$-value \\
\hline Age & $3.635 \times 10^{-4}$ & 0.1970 & -0.117 & 0.7814 \\
\%ER & $-3.535 \times 10^{-5}$ & 0.6788 & -0.169 & 0.1697 \\
Administration day & -0.002 & 0.0395 & 3.785 & 0.0301 \\
UFT (total dose) & $6.551 \times 10^{-6}$ & 0.0384 & -0.009 & 0.0570 \\
CPA (total dose) & $-1.527 \times 10^{-5}$ & 0.2649 & -0.048 & 0.0124 \\
Tumor size & -0.002 & 0.4368 & -6.109 & 0.0524 \\
DPD activity & $-1.719 \times 10^{-5}$ & 0.9004 & - & - \\
TS activity & - & - & -37.187 & 0.9004 \\
\hline
\end{tabular}

particularly to assess the effects of CPA. With respect to this issue, the present study demonstrated that UFT inhibited TS activity, but increased DPD activity. On the contrary, CPA augmented the inhibition of TS by UFT, and inhibited the DPD activity augmented by UFT. These results may clarify part of the mechanisms responsible for the combination effects of 5-FU derivatives and CPA.

TS is a key enzyme in the de novo pathway of pyrimidine synthesis, and is a very important target for chemotherapy with 5-FU or its derivatives. On the contrary, DPD degrades fluoropyrimidine to 2-fluoro- $\beta$-tetrahydrofolate $\left(5,10-\mathrm{CH}_{2} \mathrm{FH}_{2}\right)$, and is the initial and rate-limiting enzyme in this process. Fluoropyrimidines are degraded by DPD mainly in the liver, but the DPD activity in the tumor is correlated with the clinical response or survival after 5-FU-based chemotherapies in colorectal, lung and breast cancers (23-26). The present study demonstrated that in human breast cancers, NAC with UFT inhibited the TS activity in the tumor, whereas CPA augmented the inhibitory effects of UFT on TS through inhibiting DPD activity in the tumor.

In chemotherapy protocols against breast cancer, the combination of 5-FU and CPA has been widely used with other anti-cancer agents, such as doxorubicin or methotrexate. Furthermore, in other malignancies, CPA has been reported to augment the anti-tumor activity of 5-FU or its derivatives including UFT, and we have also reported the usefulness of the UC regimen against digestive organ cancers such as pancreatic cancer and ampullary cancer of the papilla of Vater (7-9). Several previous clinical reports have also suggested that this combination regimen of UFT+CPA (UC) showed anti-tumor activity against breast cancer, sometimes in combination with anthracyclines $(5,6)$. In addition, it has been reported that CPA augments the activity of RNR, which metabolizes 5-FU to fluoro-deoxyuridine monophosphate (FDUMP) in a human breast cancer xenograft line, and this may represent one of the mechanisms responsible for the beneficial effects of CPA on the anti-tumor activity of UFT (10). Other researchers have also reported that CPA enhanced the efficacy of capecitabine and 5'-deoxyfluorouridine, which are also 5-FU derivatives, by inducing TP in a human breast tumor xenograft model (11). Therefore,
CPA can modulate the activity of various enzymes, which are associated with pyrimidine metabolism. Accordingly, the present study reports for the first time that CPA inhibits the intratumoral activity of DPD, resulting in increased antitumor activity for UFT.

TS also plays a very important role in tumor growth, but previous studies have reported conflicting results with respect to the clinicopathological significance of TS. Some reports have indicated that the overexpression of TS in a tumor is a significant indicator for a poor prognosis in patients with colorectal cancer $(27,28)$, ovarian cancer (29), and gastric cancer (30-33), whereas others have reported that a high TS activity is not always associated with a poor prognosis in colon cancer (34), breast cancer (35) and gastric cancer (36). In breast cancer, it was reported that high TS levels were correlated with advanced disease stages and nodal involvement (37), but other reports have indicated no correlation $(38,39)$. Furthermore, it was reported that a high TS activity was associated with a slow disease progression (35), although another study reported that extremely high TS levels were accompanied by an unfavorable prognosis (40). In the present study, TS activity did not show any correlation with tumor size or clinical stage. Therefore, correlations between the level of TS expression and the clinicopathological factors or prognosis remain unclear.

As discussed above, TS is the main target of 5-FU or its derivatives. After 5-FU is metabolized to fluoro-deoxyuridine monophosphate (FdUMP), FdUMP forms a ternary complex with TS and folic acid, resulting in an inhibition of TS activity. With regard to the role of TS in the efficacy of 5-FU based chemotherapy, although early studies indicate that the TS inhibition was significantly correlated with the response to 5-FU $(19,41)$, the effects of the TS level on the efficacy of 5-FU or its derivatives-based chemotherapy is unclear. Many studies indicate that the low TS levels in the tumor are an indicator for favorable response to 5-FU-based chemotherapy in colorectal or gastric cancer $(30,42-44)$, whereas other reports indicate that the benefits of 5-FUbased chemotherapy are most evident in the high TS group in colorectal $(27,28)$ and breast cancer patients $(35,38)$, or that the TS levels have no effect on the efficacy of 5-FU-based 
chemotherapy in gastric $(32,33)$ and colorectal cancers (45). Therefore, this issue remains controversial.

The DPD activity increased by UFT can be inhibited by CPA. This may be one of the possible mechanisms responsible for the enhanced anti-tumor activity of 5-FU or its derivatives induced by CPA.

\section{Acknowledgements}

We gratefully acknowledge Ms. Miyuki Ishihara, Ms. Yasuko Sonoyama, and Ms. Yuka Ouchi for their assistance.

\section{References}

1. Ohta K, Taguchi T and Kimura K: Report on nationwide pooled data and cohort investigation in UFT phase II study. Cancer Chemother Pharmacol 22: 323-328, 1988.

2. Sulkes A, Benner S and Canetta R: Uracil-tegafur: An oral fluoropyrimidine active in colorectal cancer. J Clin Oncol 16: 3461-3475, 1998.

3. Van Cutsem E and Peeters M: Oral fluoropyrimidines in colorectal cancer. Semin Oncol 27 (suppl 10): 91-95, 2000.

4. Ho DH, Pazdur R, Covington W, et al: Comparison of 5fluorouracil pharmacokinetics in patients receiving continuous 5-fluorouracil infusion and oral uracil plus N1-(2'-tetrahyfrofuryl)-5-fluorouracil. Clin Cancer Res 4: 2085-2088, 1998.

5. Gregory RK, Johnston SR, Smith IE and Miles D: UFT/leucovorin plus bolus epirubicin and cyclophosphamide in advanced/metastatic breast cancer. Oncology 14: 47-49, 2000.

6. Ogawa Y, Ishikawa T, Chung SH, et al: Oral UFT and cyclophosphamide combination chemotherapy for metastatic breast cancer. Anticancer Res 23: 3453-3457, 2003.

7. Nio Y: Chemotherapy of pancreatic cancer - Adjuvant chemotherapy. Consensus Cancer Ther (in Japanese) 2: 28-31, 2003.

8. Iguchi C, Nio Y, Sato Y, et al: A case of recurrent ampullary carcinoma of the papilla of Vater in which a complete response was induced by combination therapy using radiotherapy and oral chemotherapy employing UFT and cyclophosphamide. Gan To Kagaku Ryoho (in Japanese) 26: 1641-1645, 1999.

9. Minari Y, Nio Y, Endo S, et al: A case of recurrent pancreatic cancer brought into a complete response by a multimodal treatment with intraarterial chemotherapy and radiotherapy. Gan To Kagaku Ryoho (in Japanese) 27: 121-125, 2000.

10. Haga S, Shimizu T, Imamura H, et al: Antitumor efficacy of combination chemotherapy with UFT and cyclophosphamide against human breast cancer xenografts in nude mice. Anticancer Res 19: 1791-1796, 1999.

11. Endo M, Sinbori N, Sawada N, et al: Induction of thymidine phosphorylase expression and enhancement of efficacy of capecitabine or 5'-deoxy-5-fluorouridine by cyclophosphamide in mammary tumor models. Int J Cancer 83: 127-134, 1999.

12. Van Triest B and Peters GJ: Thymidylate synthase: a target for combination therapy and determinant of chemotherapeutic response in colorectal cancer. Oncology 57: 179-194, 1999.

13. Inada T, Ogata Y, Kubota T, et al: 5-fluorouracil sensitivity and dihydropyrimidine dehydrogenase activity in advanced gastric cancer. Anticancer Res 20: 2457-2462, 2000

14. Salonga D, Danenberg KD, Johnson M, et al: Colorectal tumors responding to 5-fluorouracil have low gene expression levels of dihydropyrimidine dehydrogenase, thymidylate synthase, and thymidine phosphorylase. Clin Cancer Res 6: 1322-1327, 2000.

15. International Union Against Cancer. TNM classification of malignant tumours. 6th edition. John Wiley \& Sons, Inc., 2002.

16. NCI-CTC version 2.0. Common toxicity criteria - Notice of modifications. (http://ctep.info.nih.gov/).

17. Prasad SR, Saini S, Sumner JE, et al: Radiological measurement of breast cancer metastases to lung and liver: comparison between WHO (bidimensional) and RECIST (unidimensional) guidelines. J Comput Assist Tomogr 27: 380-384, 2003.

18. Kurosumi M, Akiyama F, Iwase T, et al: Commitee for Production of Histopathological Criteria, Japanese Breast Cancer Society. Histopathological criteria for assessment of therapeutic response in breast cancer. Breast Cancer 8: 1-2, 2001 .
19. Spears SP, Gustavsson BG, Mitchell MS, et al: Thymidylate synthase inhibition in malignant tumors and normal liver of patients given intravenous 5-fluorouracil. Cancer Res 44: 4144-4150, 1984.

20. Naguib FNM, El-Kouni MH and Cha S: Enzyme of uracil catabolism in normal and neoplastic human tissues. Cancer Res 45: 5405-5412, 1985.

21. Takechi T, Uchida J, Fujioka A and Fukushima M: Enhancing 5-fluorouracil cytotoxicity by inhibiting dihydropyrimidine dehydrogenase activity with uracil in human tumor cells. Int J Oncol 11: 1041-1044, 1997.

22. Nio Y, Sato Y, Nagami H, et al: Neoadjuvant chemotherapy of gastric cancer with oral UFT (a mixture of uracil and futrafur) during the waiting period for surgery. Anticancer Res 18: 523-530, 1998.

23. Huang CL, Yokomise H, Kobayashi S, et al: Intratumoral expression of thymidylate synthase and dihydropyrimidine dehydrogenase in non-small cell lung cancer patients treated with 5-FU-based chemotherapy. Int J Oncol 17: 47-54, 2000.

24. Horiguchi J, Takei H, Koibuchi Y, et al: Prognostic significance of dihydropyrimidine dehydrogenase expression in breast cancer. Br J Cancer 86: 222-225, 2002.

25. Ichikawa W, Uetake H, Shirota Y, et al: Combination of dihydropyrimidine dehydrogenase and thymidylate synthase gene expression in primary tumors as predictive parameters for the efficacy of fluoropyrimidine-based chemotherapy for metastatic colorectal cancer. Clin Cancer Res 9: 786-791, 2003.

26. Kakimoto M, Uetake H, Osanai T, et al: Thymidylate synthase and dihydropyrimidine dehydrogenase gene expression in breast cancer predicts 5 -FU sensitivity by a histocultural drug sensitivity test. Cancer Lett 223: 103-111, 2005.

27. Johnston PG, Fisher ER, Rockette HE, et al: The role of thymidylate synthase expression in prognosis and outcome of adjuvant chemotherapy in patients with rectal cancer. J Clin Oncol 12: 2640-2647, 1994.

28. Takenoue T, Nagawa H, Matsuda K, et al: Relation between thymidylate synthase expression and survival in colon carcinoma, and determination of appropriate application of 5fluorouracil by immunohistochemical method. Ann Surg Oncol 7: 193-198, 2000.

29. Fujiwaki R, Hata K, Nakayama K, et al: Thymidylate synthase expression in epithelial ovarian cancer: relationship with thymidine phosphorylase expression and prognosis. Oncology 59: $152-157,2000$.

30. Lenz HJ, Leichman CG, Danenberg KD, et al: Thymidylate synthase mRNA level in adenocarcinoma of the stomach: a predictor for primary tumor response and overall survival. J Clin Oncol 14: 176-182, 1996.

31. Kuniyasu T, Nakamura T, Tabuchi Y and Kuroda Y: Immunohistochemical evaluation of thymidylate synthase in gastric carcinoma using a new polyclonal antibody: the clinical role of thymidylate synthase as a prognostic indicator and its therapeutic usefulness. Cancer 83: 1300-1306, 1998.

32. Suda Y, Kuwashima Y, Tanaka Y, et al: Immunohistochemical detection of thymidylate synthase in advanced gastric cancer: a prognostic indicator in patients undergoing gastrectomy followed by adjuvant chemotherapy with 5-fluoropyrimidines. Anticancer Res 19: 805-810, 1999.

33. Tsujitani S, Konishi I, Suzuki K, et al: Expression of thymidylate synthase in relation to survival and chemosensitivity in gastric cancer patients. J Exp Clin Cancer Res 19: 189-195, 2000.

34. Umehara A, Yoshimatsu K, Endo S, et al: The correlation between the intratumoral thymidylate synthase levels and clinicopathological factors in colorectal cancer patients. J Tokyo Wom Med Uni 70: 621-629, 2000.

35. Foekens JA, Romain S, Look MP, et al: Thymidine kinase and thymidylate synthase in advanced breast cancer: response to tamoxifen and chemotherapy. Cancer Res 61: 1421-1425, 2001.

36. Choi J, Lim H, Nam DK, et al: Expression of thymidylate synthase in gastric cancer patients treated with 5-fluorouracil and doxorubicin-based adjuvant chemotherapy after curative resection. Br J Cancer 84: 186-192, 2001.

37. Ebuchi M, Sakamoto S, Kudo H, et al: Clinicopathological stages and piromidine nucleotide synthesis in human mammary carcinomas. Anticancer Res 15: 1481-1484, 1995.

38. Pestalozzi BC, Peterson HF, Gelber RD, et al: Prognostic importance of thymidylate synthase expression in early breast cancer. J Clin Oncol 15: 1923-1931, 1997. 
39. Komaki K, Kamamura Y, Ohmine Y, et al: Differences in thymidylate synthetase acitivity in involved nodes compared with primary tumor in breast cancer patients. Breast Cancer Res Treat 35: 157-162, 1995.

40. Nishimura R, Nagao K, Miyayama H, et al: Thymidylate synthase levels as a therapeutic and prognostic predictor in breast cancer. Anticancer Res 19: 5621-5626, 1999.

41. Swain SM, Lippman ME, Egan EF, et al: Fluorouracil and highdose leucovorin in previously treated patients with metastatic breast cancer. J Clin Oncol 7: 890-899, 1989.

42. Peters GJ, van der Wilt CL, van Groeningen CJ, et al: Thymidylate synthase inhibition after administration of fluorouracil with or without leucovorin in colon cancer patients: implications for treatment with fluorouracil. J Clin Oncol 12: 2035-2042, 1994.
43. Johnston PG, Lenz HJ, Leichman CG, et al: Thymidylate synthase gene and protein expression correlate and are associated with response to 5-fluorouracil in human colorectal and gastric tumors. Cancer Res 55: 1407-1412, 1995.

44. Paradiso A, Simone G, Petroni S, et al: Thymidylate synthase and p53 primary tumour expression as predictive factors for advanced colorectal cancer patients. Br J Cancer 82: 560-567, 2000 .

45. Aschele C, Debernardis D, Tunesi G, et al: Thymidylate synthase protein expression in primary colorectal cancer compared with the corresponding distant metastases and relationship with the clinical response to 5-fluorouracil. Clin Cancer Res 6: 4797-4802, 2000. 